\title{
Caracterización clínica de los casos de enfermedad meningocóccica por serogrupo W135 confirmados durante el año 2012 en Chile
}

\author{
Gabriela Moreno, Darío López, Natalia Vergara, Doris Gallegos, María F. Advis y Sergio Loayza
}

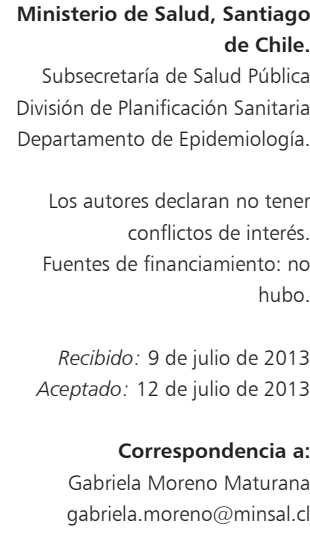

\section{Clinical characterization of cases with meningococcal disease by W135 group in Chile, 2012}

Background: During 2012 in Chile, there were 60 cases of serogroup W135 meningococcal disease, which accounts for $57.7 \%$ of identified serogroup cases. Aim: To describe main clinical features of patients with serogroup W135 meningococcal disease confirmed in 2012. Material and Methods: Descriptive study of case series based on retrospective review of medical records. Results: Male patients represented $61.7 \%$ and $46.7 \%$ were children under 5 years. At first clinical attention, $3.4 \%$ of patients were suspected of meningococcal disease, while $83.3 \%$ had meningococcemia as final diagnosis. Also at first attention, the most common symptoms or clinical signs were fever $\geq 38.0^{\circ} \mathrm{C}(60.3 \%)$, cold symptoms $(52.5 \%)$, and nausea or vomiting (46.7\%). Meningeal signs had a low frequency $(8.7 \%)$. Diarrhea was the second most common symptom found among deceased patients $(55.6 \%)$ and statistically higher than survivors $(26.8 \% ; \mathrm{p}=0.034)$. Six cases reported with sequelae: limb amputation, hearing loss or neurological damage, and mortality was $31.7 \%$. Discussion: In 2012, serogroup W135 meningococcal disease reported high mortality, atypical clinical presentation, low initial meningococcal disease diagnosis, and a high number of cases with poor clinical course.

Key words: Neisseria meningitidis, serogroup W-135, meningococcal infections, acute bacterial meningitis, meningococcal meningitis, purpura fulminans, Waterhouse-Friderichsen syndrome.

Palabras clave: Neisseria meningitidis, serogrupo W135, infecciones meningocóccicas, meningitis bacteriana aguda, meningitis meningocóccica, purpura fulminans, síndrome de Waterhouse-Friderichsen.

\section{Introducción}

$\mathrm{E}$ n la mayor parte del mundo, Neisseria meningitidis es causante de enfermedad meningocóccica (EM), principalmente en forma de meningitis y septicemia fulminante, siendo los serogrupos A, B, C responsables de $80-90 \%$ de los casos, mientras que W135 e Y lo son de $10-15 \%$ restante ${ }^{1,2}$. Todos ellos poseen el potencial de causar enfermedades endémicas y brotes epidémicos ${ }^{3,4}$. La mayor carga de EM corresponde a una zona del África subsahariana conocida como el "cinturón de la meningitis" 5 , y en el resto del mundo se presenta en pequeños conglomerados de casos y cursa con variaciones estacionales que explican una proporción variable de las epidemias por meningitis bacterianas ${ }^{6}$. En Chile, la EM es de vigilancia universal y de notificación obligatoria. Durante el período 2000-2011 se produjo un descenso en la incidencia de la enfermedad desde una tasa de 3,7 por 100.000 habitantes (hbtes.) a una de 0,4 , manteniéndose en niveles de baja endemia desde el 2006. La letalidad durante ese periodo se mantuvo en torno a $10 \%$, con excepción de los años 2010 y 2011 en que ascendió a 14,1 y $15,1 \%$, respectivamente ${ }^{8,9}$. Durante los últimos 20 años, la EM estaba asociada principalmente al serogrupo
B con una frecuencia de $78 \%$, seguido por el serogrupo C, y muy pocos casos de serogrupo W1357. Desde el 2010 el número de casos causados por serogrupo W135 aumentó progresivamente, alcanzando 21 casos el 2011 y 60 el 2012. La importancia relativa de este serogrupo se elevó desde 10 a $58 \%$ en ese lapso, desplazando al serogrupo B, fenómeno que ha sido observado en otros países de Latinoamérica ${ }^{10-12}$. Respecto a las características de la EM por serogrupo W135, los casos se concentraron en la Región Metropolitana, con predominio del sexo masculino y el grupo etario más afectado fue el de niños bajo 5 años ${ }^{1,9,12}$. Según diagnóstico clínico, para el serogrupo W135, predomina la meningococcemia por sobre la meningitis y otros diagnósticos de EM, versus el serogrupo B donde predomina el cuadro de meningitis, situación que refleja la mayor gravedad de los cuadros asociados al serogrupo W135 1 .9. En cuanto a la cepa de W135, los estudios moleculares realizados por el Instituto de Salud Pública (ISP) demuestran que la cepa pertenece al complejo clonal ST-11 y que corresponde a un linaje hipervirulento al igual que las cepas del serogrupo W135 predominantes en los últimos años en Argentina y Brasil ${ }^{11}$.

Frente a esta situación de aumento de casos por serogrupo W135, letalidad mayor y la existencia de vacuna 
específica como medida de intervención efectiva, el Ministerio de Salud (MINSAL) implementó el Plan de Acción W135 como respuesta de Salud Pública para el manejo de esta contingencia. Se formó un comité liderado por el Ministro de Salud, decretándose la alerta sanitaria. Las medidas de control incluyeron: plan de vacunación con vacuna antimeningocóccica conjugada tetravalente para A, C, W135 e Y a niños entre 9 meses y hasta 5 años de edad, plan de comunicación de riesgos y medidas preventivas, reforzamiento de la vigilancia epidemiológica y de laboratorio, actualización de la guía clínica y aseguramiento de la provisión de medicamentos para la quimioprofilaxis de los contactos ${ }^{1,13-14}$. El objetivo principal del presente estudio es describir las principales características clínicas de los casos de EM por serogrupo W135 confirmados durante el año 2012.

\section{Material y Métodos}

\section{Diseño}

Estudio de tipo descriptivo, observacional, de serie de casos.

\section{Población de estudio}

Se incluyeron en el estudio todos los casos de EM confirmadas como serogrupo W135 durante el período comprendido entre el 1 de enero y el 31 de diciembre del año 2012. Los casos fueron confirmados por el ISP y representan todos los casos del sistema público y privado en Chile.

\section{Fuentes de información}

Los datos fueron obtenidos mediante la revisión de las fichas clínicas, de las hojas de atención en servicios de urgencia de los casos y del Registro de Enfermedades de Notificación Obligatoria (ENO). La recolección de la información se realizó a través de un formulario electrónico (macro Excel) diseñado por profesionales e investigadores del Ministerio de Salud (MINSAL). Dentro de la información recogida se incluyó: antecedentes sociodemográficos (sexo, edad, región y sistema de previsión de salud), antecedentes epidemiológicos (casos primarios o secundarios, su georeferencia y tratamiento de contactos), manifestaciones clínicas, principales pruebas de laboratorio, estudio etiológico, tratamiento antimicrobiano recibido, desenlace del evento y vías de notificación utilizadas. Se recogió la información de todas las oportunidades de atención del evento de EM, incluyendo las consultas en forma ambulatoria, en los servicios de urgencias y hospitalizaciones, tanto del sistema público como del privado.

\section{Definiciones utilizadas}

En el presente estudio se utilizaron las definiciones contenidas en la Circular B51 No9 del 06/02/2009 y B51
No 50 del 05/12/2011: Caso sospechoso de EM: paciente mayor de un año de edad que presenta fiebre súbita mayor de $38,0^{\circ} \mathrm{C}$, cefalea, vómitos y al menos uno de los siguientes síntomas o signos: rigidez de nuca, alteración de conciencia, otros signos de irritación meníngea (Brudzinski o Kernig), erupción cutánea petequial o purpúrica. En los lactantes bajo un año de edad, se sospecha EM cuando la fiebre está acompañada de abombamiento de fontanela, vómitos, somnolencia, irritabilidad, convulsiones, con o sin erupción petequial. Caso confirmado de EM: caso sospechoso que se confirma por laboratorio o por nexo epidemiológico con un caso confirmado por laboratorio. Hospitalización oportuna: hospitalización dentro de las primeras $24 \mathrm{~h}$ a partir de la primera consulta. Tratamiento oportuno de los contactos: administración de quimioprofilaxis a los contactos dentro de las $24-48 \mathrm{~h}$ a partir de la hospitalización del caso $^{1,8,9,12,13}$.

\section{Análisis estadístico}

Se realizó un análisis exploratorio descriptivo. Para evaluar la distribución normal de las variables cuantitativas se utilizó el test Kolmogorov-Smirnov con una significancia de 5\%; las que cumplían con la normalidad, fueron expresadas con medias y desviación estándar (DE) y aquellas con distribución no normal, a través de medianas y percentiles. Las variables cualitativas fueron expresadas con frecuencias absolutas y porcentajes. Se realizaron comparaciones de medias en las variables continuas, mediante el test de T student o el test de U de Mann-Whitney dependiendo del criterio de normalidad para variables cuantitativas. Para evaluar la relación o asociación entre variables cuantitativas se utilizó el test de $\chi^{2}$ o el test de Fisher (este último en caso de dicotomía). Para todas las pruebas realizadas se consideró un nivel de significancia estadística de $5 \%(p<0,05)$ y se utilizó en el paquete estadístico SPSS 19.0 para Windows (SPSS, 2011).

\section{Marco ético y regulatorio}

Se revisaron las normas de confidencialidad de las fichas clínicas contenidas en el artículo 13 de la ley $\mathrm{N}^{\mathrm{o}}$ $20.584^{15}$, las normas del Código Sanitario y del artículo $4^{\circ}$ del DFL No 1 del año $2005^{16}$ del MINSAL, en el cual se ordena a la Subsecretaría de Salud Pública efectuar la vigilancia en Salud Pública, evaluar la situación de salud de la población y, velar por el debido cumplimiento de los objetivos en materia de salud. Se consultó además a expertos de bioética y a la División de Jurídica del MINSAL y se decidió que, en el contexto de una alerta sanitaria, no existía un impedimento de orden jurídico ni ético para llevar a cabo el estudio basado en la revisión de las fichas clínicas por parte de los investigadores. Lo señalado anteriormente se encuentra también recogido en el artículo 10 del reglamento sobre fichas clínicas, decreto 
Tabla 1. Características epidemiológicas y sociodemográficas de los casos de EM por serogrupo W135. Chile, año 2012

\begin{tabular}{|c|c|c|c|c|c|c|c|}
\hline & & \multicolumn{2}{|c|}{$\begin{array}{l}\text { Fallecidos } \\
(\mathrm{n}=19)\end{array}$} & \multicolumn{2}{|c|}{$\begin{array}{l}\text { Vivos } \\
(n=41)\end{array}$} & \multicolumn{2}{|c|}{$\begin{array}{c}\text { Total } \\
(n=60)\end{array}$} \\
\hline & & $\mathrm{n}$ & $\%$ & $\mathrm{n}$ & $\%$ & $\mathrm{n}$ & $\%$ \\
\hline \multirow[t]{2}{*}{ Sexo } & Hombre & 11 & 57,9 & 26 & 63,4 & 37 & 61,7 \\
\hline & Mujer & 8 & 42,1 & 15 & 36,6 & 23 & 38,3 \\
\hline \multirow[t]{6}{*}{ Grupos de edad } & 0 a 8 meses & 2 & 10,5 & 8 & 19,5 & 10 & 16,7 \\
\hline & 9 meses a 4 años & 3 & 15,8 & 15 & 36,6 & 18 & 30,0 \\
\hline & 5 a 9 años & 0 & 0,0 & 4 & 9,8 & 4 & 6,7 \\
\hline & 10 a 19 años & 2 & 10,5 & 1 & 2,4 & 3 & 5,0 \\
\hline & 20 a 60 años & 8 & 42,1 & 9 & 22,0 & 17 & 28,3 \\
\hline & > 60 años & 4 & 21,1 & 4 & 9,8 & 8 & 13,3 \\
\hline \multirow[t]{8}{*}{ Región } & Arica y Parinacota & 0 & 0,0 & 1 & 2,4 & 1 & 1,7 \\
\hline & Tarapacá & 0 & 0,0 & 1 & 2,4 & 1 & 1,7 \\
\hline & Antofagasta & 1 & 5,3 & 0 & 0,0 & 1 & 1,7 \\
\hline & Valparaíso & 2 & 10,5 & 2 & 4,9 & 4 & 6,7 \\
\hline & Metropolitana & 14 & 73,7 & 34 & 82,9 & 48 & 80,0 \\
\hline & O'Higgins & 0 & 0,0 & 1 & 2,4 & 1 & 1,7 \\
\hline & Araucanía & 1 & 5,3 & 1 & 2,4 & 2 & 3,3 \\
\hline & Los Lagos & 1 & 5,3 & 1 & 2,4 & 2 & 3,3 \\
\hline \multirow{3}{*}{$\begin{array}{l}\text { Previsión de sa- } \\
\text { lud }\end{array}$} & FONASA* & 14 & 73,7 & 36 & 87,8 & 50 & 83,3 \\
\hline & ISAPRE** & 4 & 21,1 & 3 & 7,3 & 7 & 11,7 \\
\hline & DIPRECA*** & 0 & 0,0 & 1 & 2,4 & 1 & 1,7 \\
\hline \multirow{10}{*}{$\begin{array}{l}\text { Antecedentes } \\
\text { mórbidos }\end{array}$} & Mieloma múltiple & 1 & 5,9 & 1 & 2,6 & 2 & 3,6 \\
\hline & Asplenia & 0 & 0,0 & 1 & 2,6 & 1 & 1,8 \\
\hline & Infección por VIH & 0 & 0,0 & 1 & 3,1 & 1 & 2,6 \\
\hline & $\begin{array}{l}\text { Infección reciente por } S \text {. } \\
\text { pneumoniae }\end{array}$ & 0 & 0,0 & 1 & 2,6 & 1 & 1,8 \\
\hline & $\begin{array}{l}\text { Infección reciente por } H \text {. } \\
\text { influenzae b }\end{array}$ & 0 & 0,0 & 1 & 2,6 & 1 & 1,8 \\
\hline & Insuficiencia renal & 2 & 11,8 & 4 & 10,3 & 6 & 10,7 \\
\hline & Cardiovasculares & 9 & 52,9 & 7 & 17,9 & 16 & 28,6 \\
\hline & Diabetes mellitus & 4 & 25,0 & 4 & 11,1 & 8 & 15,4 \\
\hline & Obesidad & 2 & 12,5 & 6 & 16,2 & 8 & 15,1 \\
\hline & Tabaquismo activo**** & 3 & 33,3 & 2 & 15,4 & 5 & 22,7 \\
\hline $\begin{array}{l}\text { Quimioprofilaxis } \\
\text { de contactos }\end{array}$ & Sí & 18 & 94,7 & 40 & 97,6 & 58 & 96,7 \\
\hline
\end{tabular}

*FONASA: Fondo Nacional de Salud. **ISAPRE: Instituciones de Salud Previsional. ***DIPRECA: Dirección de Previsión de Carabineros. ****Tabaquismo activo: se consideró a los pacientes sobre 12 años. Información registrada en 21 pacientes de 27. La exposición pasiva al humo ambiental del tabaco no se registró en la ficha clínica.

\begin{tabular}{|c|c|c|c|c|}
\hline \multicolumn{5}{|c|}{ Tabla 1B. Letalidad por grupo etario } \\
\hline \multirow{6}{*}{ Edad } & & n de fallecidos & $\mathrm{n}$ casos & Letalidad (\%) \\
\hline & Menores de 5 años & 5 & 28 & 17,9 \\
\hline & 5 a 19 años & 2 & 7 & 28,6 \\
\hline & 20 a 60 años & 8 & 17 & 47,1 \\
\hline & Mayores de 60 años & 4 & 8 & 50,0 \\
\hline & Total & 19 & 60 & 31,7 \\
\hline
\end{tabular}

$\mathrm{N}^{\circ} 41$ del año 2012 del MINSAL, el cual está plenamente vigente (Memo A15/4310 del 04 de diciembre 2012: Uso de fichas clínicas). Se redactó un ordinario formal desde la Subsecretaría de Salud Pública dirigido a los directores de cada servicio de salud para la petición de las copias de las fichas clínicas y posteriormente, con el objetivo de resguardar la seguridad y confidencialidad de la información de cada paciente, se estableció un sistema con recepción firmada de las mismas en el MINSAL y almacenamiento bajo llave con acceso restringido a los investigadores principales del estudio.

\section{Resultados}

\section{Antecedentes sociodemográficos y epidemiológicos}

Durante el 2012 se confirmó un total de 134 casos de EM, de los cuales 60 correspondieron al serogrupo W135 (Tabla 1A). El 46,7\% tenía menos de 5 años de edad, seguidos por los de 60 años y más, y el grupo entre 40 y 60 años, con $15,0 \%$ cada uno. Predominó el sexo masculino $(61,7 \%)$; la tasa de ataque en los hombres fue de 0,4 por cien mil hbtes. y en mujeres fue de 0,3 por cien mil hbtes. Las tasas de ataque según edad fueron las siguientes: menor de 9 meses 6,0 por cien mil hbtes, 9 meses a menores de 5 años 1,7 por cien mil hbtes, de 5 años a menores de 10 años 0,3 por cien mil hbtes, de 10 años a menores de 20 años 0,1 por cien mil hbtes, de 20 años a menores de 60 años 0,2 hbtes, 60 o más años 0,4 por cien mil hbtes y la tasa país 0,3 por cien mil hbtes. La letalidad fue de $31,7 \%$ (19 casos fallecidos de 60). El grupo con mayor letalidad fue el de mayores de 60 años $(50,0 \%)$, seguido por los de 20 a 60 años $(47,1 \%)$, en tanto que la menor letalidad se observa en los menores de 5 años $(17,9 \%)$ (Tabla 1B). En 8 de las 15 regiones del país, se presentaron casos, donde la Región Metropolitana (RM) concentró $80,0 \%$ (48 casos), seguida por la Región de Valparaíso con $6,7 \%$. En 27 de las 52 comunas de la RM se presentaron casos y todos ellos fueron geo-referenciados (Figura 1). La previsión de salud correspondió a FONASA (Fondo Nacional de Salud: seguro de salud estatal) en $83,3 \%$ y a ISAPRE (Instituciones de Salud Previsional: seguro privado de salud) en $11,7 \%$ de los casos. Respecto a los antecedentes mórbidos, destaca la presencia de al menos un antecedente cardiovascular en 16 casos $(28,6 \%)$, hecho que fue estadísticamente superior en los fallecidos $(52,9$ versus $17,9 \% ; \mathrm{p}=0,008)$ y en el grupo etario de 20 años y más $(81,3$ versus $18,7 \% ; p=0,006)$. Otros antecedentes fueron tabaquismo activo $(22,7 \%)$ y diabetes mellitus $(15,4 \%)$, ambos presentes en mayor frecuencia en el grupo de los fallecidos. Obesidad en cambio, fue mayor en los sobrevivientes $(15,1 \%)$, sin encontrarse diferencias estadísticamente significativas según desenlace. Todos los casos fueron casos primarios, no hubo casos secundarios. 
La quimioprofilaxis de los contactos se realizó con rifampicina o ciprofloxacina, siguiendo la normativa contenida en la Circular B51 No9 del 06/02/2009 en 58 de los 60 casos (96,7\%). Dos casos no recibieron quimioprofilaxis pues fueron diagnosticados por hallazgo de laboratorio y estaban fuera del plazo de oportunidad.

\section{Caracterización clínica de la primera consulta}

En la primera consulta los síntomas y signos más frecuentes registrados en la ficha clínica, fueron: fiebre $\geq 38,0^{\circ} \mathrm{C}(60,3 \%)$ como dato objetivo medido al momento del examen, catarro respiratorio $(52,5 \%)$, náuseas y/o vómitos $(46,7 \%)$, anorexia $(39,0 \%)$ y diarrea $(35,6 \%)$, en tanto, los signos de irritación meníngea aparecieron con una frecuencia de $8,7 \%$, en el total de casos (rigidez de nuca: 6,9\% y signo de Brudzinski: 1,8\%). La fiebre en la anamnesis también fue analizada: 57 de los 60 casos refirieron presentar fiebre $(95,0 \%)$ y 39 de éstos, refirieron fiebre cuantificada igual o mayor a $38,0^{\circ} \mathrm{C}(65,0 \%)$, sin diferencias estadísticamente significativas entre sobrevivientes y fallecidos (Figura 2). La diarrea fue el segundo síntoma más frecuente $(55,6 \%)$, y es el único síntoma que presentó diferencia estadísticamente significativa respecto de los sobrevivientes (26,8\%); p: 0,034. Se llevó a cabo un sub-análisis de los cinco síntomas y signos más frecuentes en la primera consulta por desenlace final, sexo y edad, sin encontrarse diferencias estadísticamente significativas entre ambos grupos (datos no mostrados).

\section{Diagnóstico en la primera consulta}

Los diagnósticos iniciales fueron agrupados en ocho grandes grupos, de los cuales destacan como los más frecuentes: fiebre $\sin$ foco $(27,6 \%)$, infecciones gastrointestinales $(24,1 \%)$ y sepsis $(20,7 \%)$. La EM fue planteada en sólo 3,4\% de los casos y corresponden a dos pacientes que sobrevivieron (Figura 3).

\section{Origen y destino después de la primera consulta}

La primera consulta se originó en Unidades de Emergencia Hospitalaria (UEH) en 46,7 \% de los casos (28 casos), seguida por Servicios de Atención Primaria de Urgencia (SAPU) en 28,3\% (17 casos) y por servicios de urgencia privados en 25,0\% (15 casos). Después de la primera consulta, el destino de los casos fue a la hospitalización en 45,0\% (27 casos), al domicilio en 33,3\% (20 casos), y derivado a otro establecimiento de mayor complejidad en 18,3\% (11 casos). De este último grupo, ocho casos finalmente se hospitalizaron. Al analizar origen y destino según desenlace, destaca que de los 17 casos atendidos en SAPU, cuatro fueron derivados a domicilio y posteriormente fallecieron $(36,4 \%)$, en cambio de los 28 que consultaron en UEH, un paciente fue derivado a domicilio y posteriormente falleció y de los 16 pacientes que consultaron en servicios de urgencia privados, un paciente fue dado de alta a domicilio y falleció. Dos pacientes fallecieron en la primera consulta y no alcanzaron a ser hospitalizados, uno en SAPU y el otro en UEH (Tabla 2). De los 60 casos, 34 se hospitalizaron finalmente después de la primera consulta $(58,6 \%)$ y cuando los pacientes fueron internados, el servicio de hospitalización correspondió a UTI/UCI en 58,6\%, Pediatría en $29,3 \%$

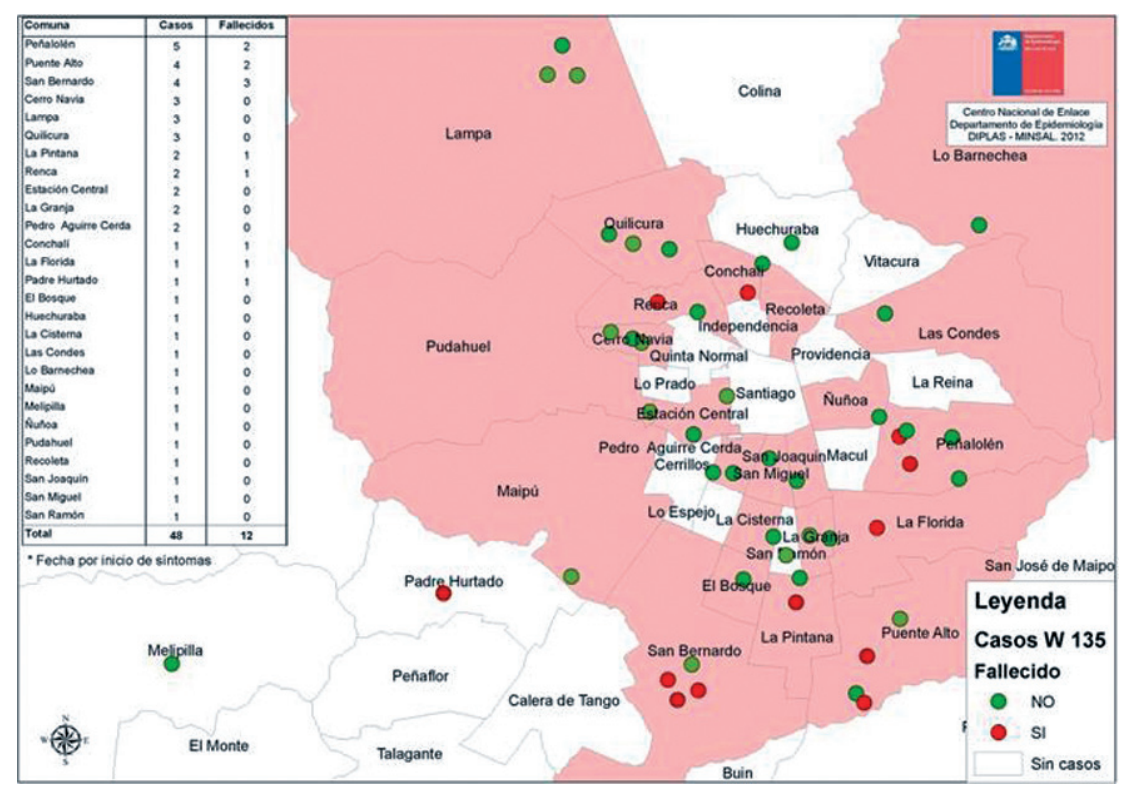

Figura 1.Geo-referenciación de los casos vivos y fallecidos por EM serogrupo W135 de la Región Metropolitana, durante el año 2012. Fuente: Centro Nacional de Enlace, Departamento de Epidemiología, Ministerio de Salud de Chile, 2012.

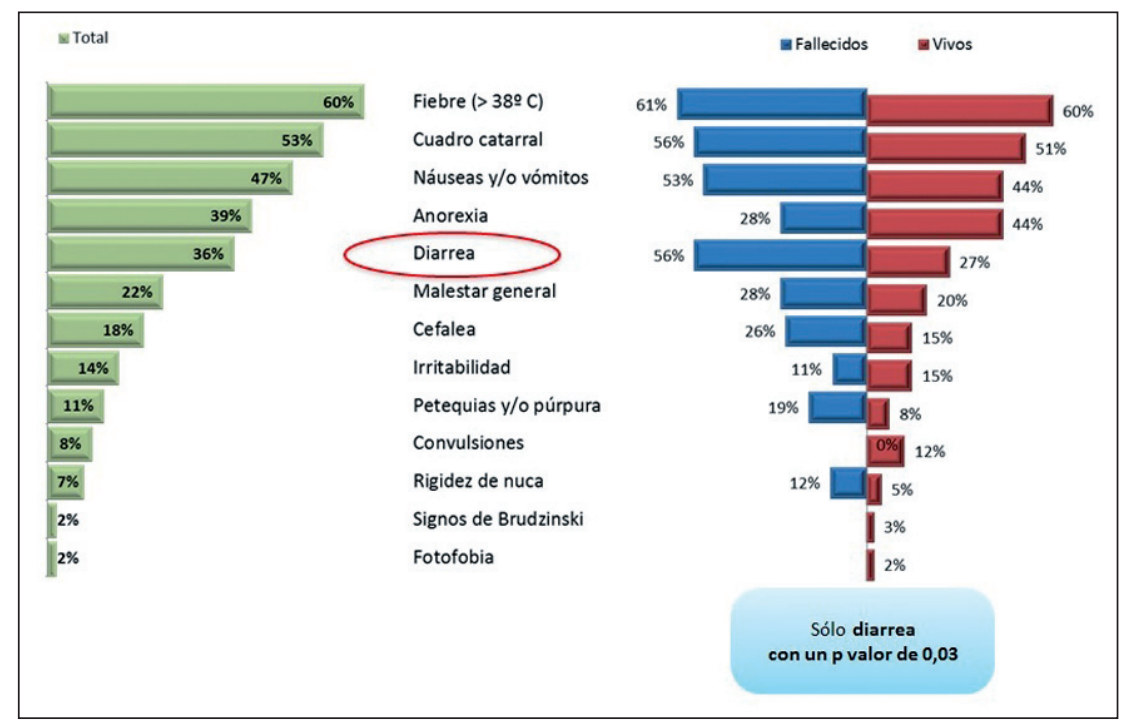

Figura 2.Distribución porcentual de la presencia de signos y síntomas en la primera consulta médica de los casos de EM por serogrupo W135 durante el año 2012. A la izquierda la distribución porcentual de síntomas y signos del total de casos ordenados de mayor a menor frecuencia, y a la derecha la distribución porcentual comparativa de síntomas y signos de fallecidos y sobrevivientes. La diarrea se presentó con una diferencia estadísticamente significativa entre ambos grupos. Cuadro catarral fue definido como la presencia de coriza, odinofagia y tos seca. 
Figura 3. Distribución en frecuencias absolutas de los diagnósticos sindromáticos y/o diagnósticos agrupados en la primera consulta de los casos de EM por serogrupo W135 durante el año 2012. Chile.

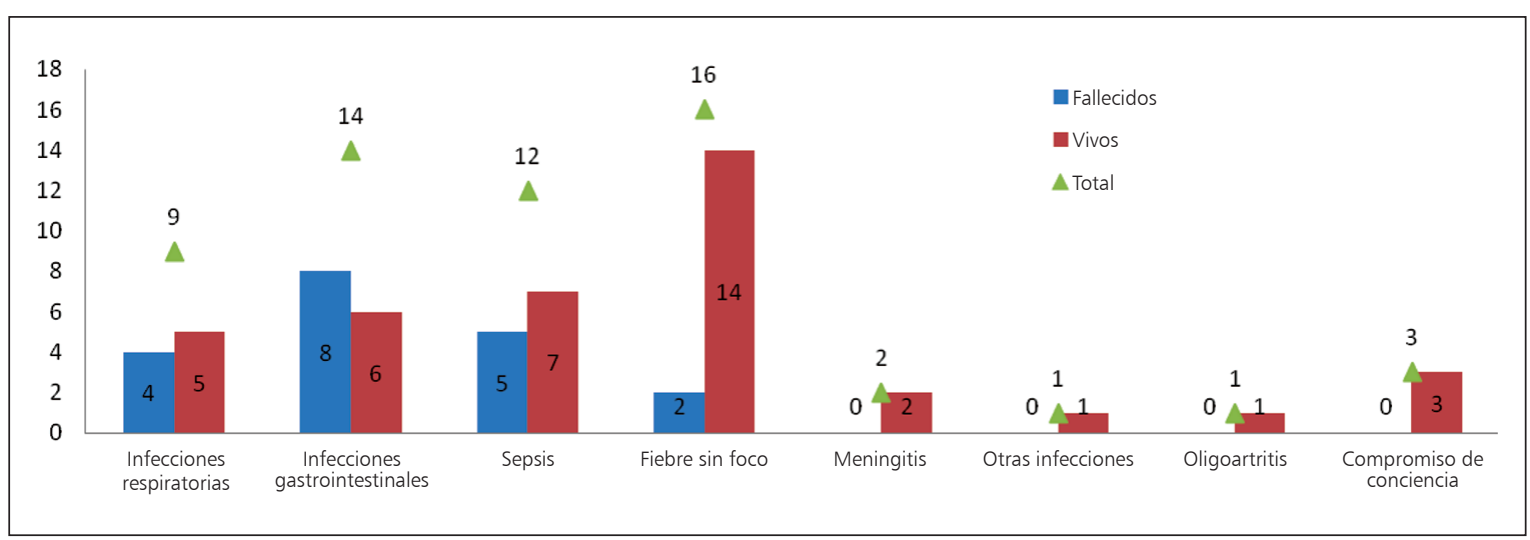

\begin{tabular}{|c|c|c|c|c|c|c|c|c|c|}
\hline & & & & \multicolumn{2}{|c|}{ Fallecidos } & \multicolumn{2}{|c|}{ Vivos } & \multicolumn{2}{|c|}{ Total } \\
\hline & & & & $\mathbf{n}$ & $\%$ & $\mathbf{n}$ & $\%$ & $\mathbf{n}$ & $\%$ \\
\hline \multirow{11}{*}{ Origen } & \multirow{3}{*}{ SAPU* } & \multirow{11}{*}{ Destino } & Domicilio & 4 & 36,4 & 4 & 66,7 & 8 & 47,1 \\
\hline & & & Derivado a otro establecimiento & 6 & 54,5 & 2 & 33,3 & 8 & 47,1 \\
\hline & & & Fallece & 1 & 9,1 & & & 1 & 5,9 \\
\hline & \multirow{4}{*}{$\mathrm{UEH}^{* *}$} & & Domicilio & 1 & 16,7 & 9 & 40,9 & 10 & 35,7 \\
\hline & & & Derivado a otro establecimiento & 1 & 16,7 & 1 & 4,5 & 2 & 7,1 \\
\hline & & & Hospitalización & 3 & 50,0 & 12 & 54,5 & 15 & 53,6 \\
\hline & & & Fallece & 1 & 16,7 & & & 1 & 3,6 \\
\hline & \multirow{4}{*}{$\begin{array}{l}\text { Clínica/ } \\
\text { consulta privada }\end{array}$} & & Domicilio & 1 & 50,0 & 1 & 7,7 & 2 & 13,3 \\
\hline & & & Derivado a otro establecimiento & 1 & 50,0 & 0 & 0,0 & 1 & 6,7 \\
\hline & & & Hospitalización & 0 & 0,0 & 12 & 92,3 & 12 & 80,0 \\
\hline & & & Fallece & 0 & 0,0 & & & 0 & 0,0 \\
\hline
\end{tabular}

y Medicina/Cirugía en $12,0 \%$. Sin embargo, $69,0 \%$ de los casos requirió ingreso en UCI, UTI o alguna unidad similar en algún momento de su hospitalización.

\section{Caracterización clínica en la primera hospitalización}

Los síntomas y signos más frecuentes al momento del ingreso hospitalario fueron: compromiso del estado general $(80,4 \%)$, catarro respiratorio $(53,6 \%)$, taquicardia $(45,3 \%)$, fiebre $\geq 38,0^{\circ} \mathrm{C}(41,8 \%)$ y anorexia $(33,9 \%)$; en cambio, signos de irritación meníngea se presentaron en $27,9 \%$ de los pacientes (rigidez de nuca: $14,5 \%$, signo de Kernig: 7,5\% y signo de Brudzinski: 5,9\%). Catarro respiratorio y petequias $\mathrm{y} / \mathrm{o}$ púrpura se presentaron en mayor frecuencia y con una diferencia estadísticamente significativa en los fallecidos (p: 0,001 y p: 0,006 respectivamente), en cambio anorexia e irritabilidad (en niños bajo 5 años) se presentaron con mayor frecuencia en los sobrevivientes (p: 0,021 y p: 0,020 , respectivamente) (Figura 4). En 30,0\% de los casos se planteó el diagnóstico de EM al momento del ingreso hospitalario y seguido por $21,7 \%$ de los casos en los cuales se planteó el diagnóstico de sepsis. Otros diagnósticos fueron: bronconeumonía, escarlatina, infección urinaria, fiebre tifoidea, gastroenteritis aguda, influenza, artritis séptica, celulitis y síndrome febril.

\section{Diagnóstico final}

En relación al diagnóstico realizado al final de hospitalización, se consideró el diagnóstico de notificación final porque posee una mayor precisión al incluir el cuadro clínico, las pruebas de laboratorio y la evolución del caso. La presentación clínica más frecuente fue la meningococcemia en $83,3 \%$ (meningococcemia: $71,7 \%$; meningitis y meningococcemia: $10,0 \%$ y síndrome de Waterhouse-Friderichsen: un caso), mientras que meningitis como forma pura de presentación ocupó el 15,0\%. Un caso se manifestó como artritis séptica (Figura 5). El análisis de los casos con diagnósticos relacionados con meningococcemia, muestra que $89,5 \%$ de los fallecidos 
(17 de 19 casos) y 80,5\% de los sobrevivientes (33 de 41 casos) tuvieron esta presentación clínica, lo cual no fue estadísticamente significativo. Con esta misma variable tampoco hubo diferencia por sexo y edad. El análisis de los casos de meningitis muestra que $10,5 \%$ de los fallecidos y $17,1 \%$ de los sobrevivientes tuvieron esta presentación y tampoco hubo diferencias estadísticamente significativas entre sobrevivientes y fallecidos, por sexo y edad.

\section{Temporalidad de los casos}

Se definieron seis períodos para analizar la evolución temporal de los casos. Entre los primeros síntomas y la primera consulta, $80,0 \%$ tardó dos días o menos en consultar por primera vez. Entre la primera consulta y la primera hospitalización, 86,2\% se hospitalizó en las primeras 24 h. En ambos períodos no hubo diferencias estadísticamente significativas entre sobrevivientes y fallecidos (Tabla 3A). Respecto de los decesos, 64,7\% de los casos falleció a un día o menos de haber sido hospitalizado y al analizar el período desde el inicio de los síntomas y el fallecimiento, el 47,4\% murió entre uno y dos días después de haber iniciado síntomas (Tabla 3B). Dos pacientes no fueron considerados en este análisis porque fallecieron antes de indicarse la hospitalización. Respecto a la estadía hospitalaria, 72,4\% de los casos estuvo internado por un período de cinco o más días. Del $27,6 \%$ restante (16 pacientes), 13 fallecieron y 3 sobrevivieron; estos últimos presentaron una hospitalización breve, en uno de ellos se decidió un alta precoz debido a la buena evolución y respuesta al tratamiento, y en los otros dos casos el diagnóstico se realizó con posterioridad al egreso mediante hallazgo de laboratorio; ambos cursaron con buena evolución. Trece de 17 pacientes hospitalizados y fallecidos $(76,5 \%)$, tuvieron una estadía hospitalaria de cuatro días o menos, en comparación con los tres pacientes hospitalizados y que sobrevivieron $(7,3 \%)$, diferencia estadísticamente significativa $(p<0,05)$. El período entre el inicio de los síntomas y la primera dosis de antimicrobiano se relaciona con la prontitud del inicio del tratamiento antimicrobiano, observándose que 57,9\% de los casos recibió su primera dosis en los dos primeros días de iniciados los primeros síntomas, y 10,5\% en seis o más días. No se observaron diferencias estadísticamente significativas entre sobrevivientes y fallecidos.

\section{Exámenes de laboratorio}

Las principales alteraciones encontradas en las pruebas de laboratorio realizadas al ingreso del paciente, fueron las transaminasas hepáticas elevadas en 70,8\% (p>0,05), trombocitopenia en $62,7 \%$, creatininemia alta en $55,3 \%$, leucocitosis/leucopenia en 37,7/28,3\%, p $<0,05$ para estos tres parámetros entre fallecidos y vivos. La proteína $\mathrm{C}$ reactiva-PCR obtenida dentro de los primeros tres días de iniciados los síntomas (48 casos) fue $>5,0 \mathrm{mg} / \mathrm{L}$ en $95,8 \%$. La media fue de $171,5 \mathrm{mg} / \mathrm{L}$, con un mínimo de 2,3 y un máximo de $466,7 \mathrm{mg} / \mathrm{L}$. No se observaron diferencias estadísticamente significativas entre fallecidos y sobrevivientes (Tabla 4). En relación al estudio etiológico, $N$. meningitidis fue aislada en muestras de hemocultivos (75,0\%, 45 casos), LCR (18,3\%, 11 casos), LCR y hemocultivo en dos casos, líquido articular y hemocultivo en un caso y líquido articular en un caso.

\section{Tratamiento recibido}

Recibieron tratamiento antimicrobiano 58 de los 60 casos $(96,7 \%)$, los dos pacientes que no recibieron, co-

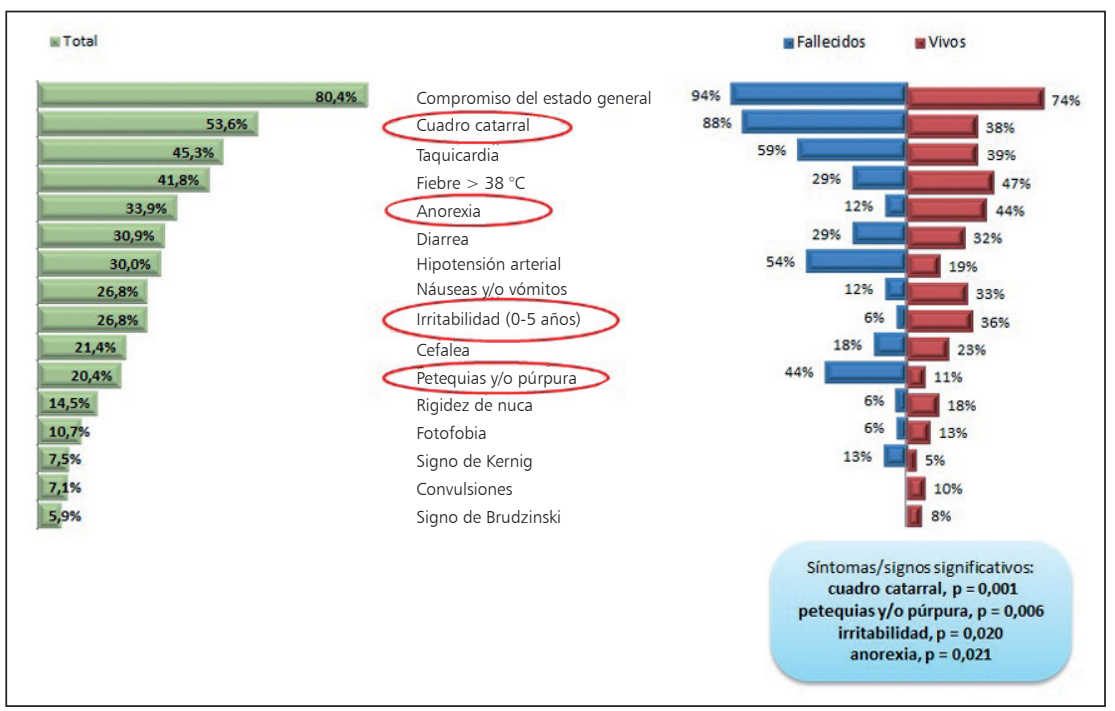

Figura 4. Distribución porcentual de la presencia de síntomas y signos en la primera hospitalización de los casos de EM por serogrupo W135 del año 2012. Chile. A la izquierda la distribución porcentual de síntomas y signos del total de casos ordenados de mayor a menor, y a la derecha la distribución porcentual comparativa de síntomas y signos de fallecidos y sobrevivientes. Cuadro catarral, anorexia, irritabilidad y petequias y/o púrpura presentan diferencias estadísticamente significativas entre ambos grupos. Los valores de referencia utilizados para frecuencia cardíaca y presión arterial fueron los de la American Heart Association.

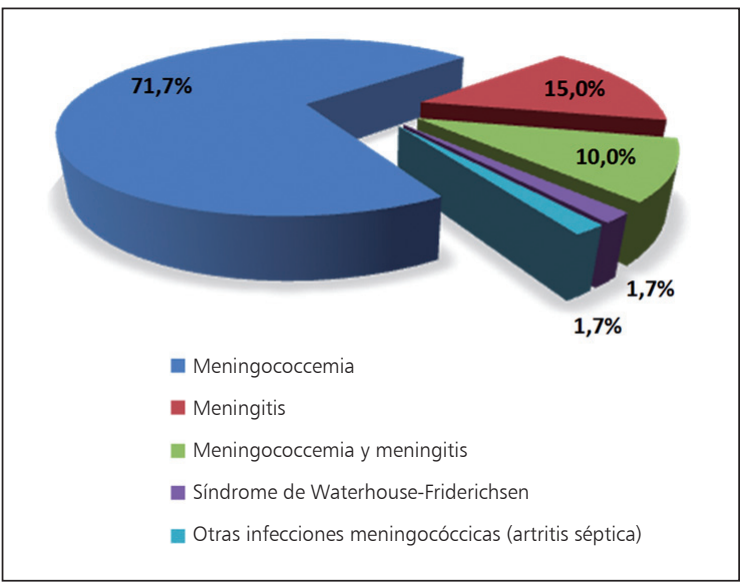

Figura 5. Distribución porcentual de los diagnósticos de notificación final de los casos de EM por serogrupo W135 durante el año 2012 Chile. 
Tabla 3. Temporalidad de los casos vivos y fallecidos de EM por serogrupo W135, año 2012

\begin{tabular}{|c|c|c|c|c|c|c|c|c|}
\hline \multicolumn{9}{|c|}{ Tabla 3A. Vivos y fallecidos } \\
\hline & & \multicolumn{2}{|c|}{$\begin{array}{l}\text { Fallecidos } \\
(\mathrm{n}=19)\end{array}$} & \multicolumn{2}{|c|}{$\begin{array}{c}\text { Vivos } \\
(n=41)\end{array}$} & \multicolumn{2}{|c|}{$\begin{array}{c}\text { Total } \\
(n=60)\end{array}$} & \multirow[t]{2}{*}{$\begin{array}{c}p \\
\text { valor }\end{array}$} \\
\hline & & $\mathbf{n}$ & $\%$ & n & $\%$ & n & $\%$ & \\
\hline \multirow{3}{*}{$\begin{array}{l}\text { Lapso entre: } \\
\text { - primeros síntomas y } \\
\text { primera consulta }\end{array}$} & 0-2 días & 14 & 73,7 & 34 & 82,9 & 48 & 80,0 & \multirow[t]{3}{*}{0,583} \\
\hline & 3-5 días & 2 & 10,5 & 4 & 9,8 & 6 & 10,0 & \\
\hline & $\geq 6$ días & 3 & 15,8 & 3 & 7,3 & 6 & 10,0 & \\
\hline \multirow{3}{*}{$\begin{array}{l}\text { - primera consulta y } \\
\text { primera hospitalización }\end{array}$} & 0-1 día & 16 & 94,1 & 34 & 82,9 & 50 & 86,2 & \multirow[t]{3}{*}{0,480} \\
\hline & 2-3 días & 1 & 5,9 & 5 & 12,2 & 6 & 10,3 & \\
\hline & 4-5 días & 0 & 0,0 & 2 & 4,9 & 2 & 3,4 & \\
\hline \multirow{4}{*}{$\begin{array}{l}\text { Duración de estadía } \\
\text { hospitalaria }\end{array}$} & 0-4 días & 13 & 76,5 & 3 & 7,3 & 16 & 27,6 & \multirow[t]{4}{*}{0,000} \\
\hline & 5-9 días & 1 & 5,9 & 17 & 41,5 & 18 & 31,0 & \\
\hline & 10-14 días & 0 & 0,0 & 9 & 22,0 & 9 & 15,5 & \\
\hline & $\geq 15$ días & 3 & 17,6 & 12 & 29,3 & 15 & 25,9 & \\
\hline \multirow{3}{*}{$\begin{array}{l}\text { Lapso entre: primeros } \\
\text { síntomas y primera dosis } \\
\text { de antimicrobiano }\end{array}$} & 0-2 días & 10 & 62,5 & 23 & 56,1 & 33 & 57,9 & \multirow[t]{3}{*}{0,263} \\
\hline & 3-5 días & 3 & 18,8 & 15 & 36,6 & 18 & 31,6 & \\
\hline & $\geq 6$ días & 3 & 18,8 & 3 & 7,3 & 6 & 10,5 & \\
\hline
\end{tabular}

\begin{tabular}{|llcc|}
\hline & \multicolumn{2}{c|}{ Tabla 3B. Fallecidos } & \\
\hline & & \multicolumn{2}{c|}{ Fallecidos $(\mathbf{n}=\mathbf{1 9})$} \\
Lapso entre: & & $\mathbf{n}$ & $\%$ \\
- Hospitalización y defunción & 0-1 día & 11 & 64,7 \\
& $2-3$ días & 1 & 5,9 \\
& $\geq 4$ días & 5 & 29,4 \\
- Primeros síntomas y defunción & $1-2$ días & 9 & 47,4 \\
& 3-4 días & 3 & 15,8 \\
& $\geq 5$ días & 7 & 36,8 \\
\hline
\end{tabular}

Tabla 4. Valores de PCR* de ingreso de los casos de EM por serogrupo W135. Chile, año 2012

\begin{tabular}{|c|c|c|c|c|c|c|}
\hline & & & & & & $p$ valor \\
\hline & $0-5$ & 1 & 7,7 & 1 & 2,9 & \\
\hline & $6-100$ & 5 & 38,5 & 12 & 34,3 & \\
\hline Rangos de PCR & $101-200$ & 3 & 23,3 & 6 & 17,1 & $>0,05$ \\
\hline$(\mathrm{mg} / \mathrm{L})$ & 201-300 & 2 & 15,4 & 9 & 25,7 & \\
\hline & $301-400$ & 0 & 0,0 & 7 & 20,0 & \\
\hline & $>401$ & 2 & 15,4 & 1 & 0,0 & \\
\hline
\end{tabular}

rresponden a dos pacientes que fallecieron precozmente. En 55 de los 58 casos $(94,8 \%)$ fueron indicadas cefalosporinas de tercera generación, principalmente ceftriaxona y cefotaxima. Otros antimicrobianos indicados fueron vancomicina en 20 casos $(37,0 \%)$, penicilina $G$ sódica en 10 casos $(18,5 \%)$ y ampicilina en tres casos $(5,9 \%)$. La duración de la antibioterapia, tuvo una media de 4,0 días en los fallecidos y de 10,1 días en los sobrevivientes, siendo estadísticamente significativa la diferencia entre vivos y fallecidos (Tabla 5). Fármacos vasoactivos se indicaron en $49,0 \%$ de los casos, siendo estadísticamente superior en los fallecidos. El 55,6\% recibió corticosteroides, siendo también mayor su indicación en los fallecidos (Tabla 5).

\section{Complicaciones y secuelas}

En los fallecidos, las tres complicaciones más frecuentes registradas fueron: shock séptico $(36,4 \%$, cuatro casos), falla multiorgánica ( $18,2 \%$, dos casos) y púrpura fulminante (18,2\%, dos casos). En cambio en los sobrevivientes, las principales complicaciones registradas fueron: shock séptico (48,0\%, 12 casos), neumonía nosocomial (16,0\%, cuatro casos) y otras infecciones nosocomiales (16,0\%, cuatro casos). Seis pacientes presentaron secuelas: déficit motor en dos casos, hipoacusia en dos casos y amputación de las cuatro extremidades en dos casos, uno de los cuales falleció.

\section{Discusión}

Las dificultades del estudio se relacionaron con la obtención de las fichas, envío incompleto de la información, datos clínicos registrados en forma heterogénea y poca legibilidad. Lo anterior pudo conllevar sesgos de información producto de la recolección incompleta de los datos y, sesgos del observador debido a que los criterios y herramientas clínicas dependen del conocimiento y sospecha de la EM. Se intentó minimizar ambos sesgos con la utilización de las siguientes fuentes de información: fichas clínicas, hojas de atención en servicios de urgencia de los casos, formulario de notificación inmediata y Registro ENO y con la revisión paralela de estas fuentes por dos investigadores cuando se presentaron dudas en la interpretación de los datos. No obstante estas limitaciones, el aporte de este estudio es que los resultados aquí expuestos, representan un primer acercamiento al conocimiento de los casos de EM por W135, serogrupo emergente en Chile, y facilita la comparación del comportamiento clínico con otros países.

En Chile, el aumento de casos de EM por serogrupo W135 durante el año 2012 se caracterizó por tener una elevada tasa de letalidad (31,7\%), se presentó en ocho regiones del país, concentrándose $80 \%$ en la RM y $6,7 \%$ en la Región de Valparaíso. Los grupos más afectados fueron hombres, y el grupo etario de 0-5 años, teniendo el grupo bajo 9 meses de edad la mayor tasa de ataque, seguido del grupo de 9 meses a niños bajo 5 años. El cuadro clínico de la EM invasora, según varios estudios, es un proceso dinámico y se presenta en un amplio espectro clínico que puede ir desde un síndrome febril hasta un cuadro de meningitis, meningococcemia o una combinación de am- 


\begin{tabular}{|c|c|c|c|c|c|c|c|}
\hline \multirow[b]{3}{*}{ Fármacos } & \multicolumn{2}{|c|}{ Fallecidos $(n=19)$} & \multicolumn{2}{|c|}{ Vivos $(n=41)$} & \multicolumn{2}{|c|}{ Total $(n=60)$} & \multirow[t]{2}{*}{ p valor } \\
\hline & n & $\%$ & $\mathrm{n}$ & $\%$ & $\mathrm{n}$ & $\%$ & \\
\hline & 17 & 89,5 & 41 & 100,0 & 58 & 96,7 & 0,097 \\
\hline \multicolumn{8}{|l|}{ Antimicrobianos } \\
\hline Cefalosporinas* & 16 & 100,0 & 39 & 95,1 & 55 & 96,5 & 1,000 \\
\hline Vancomicina & 9 & 60,0 & 11 & 28,2 & 20 & 37,0 & 0,030 \\
\hline Penicilina & 2 & 13,3 & 8 & 20,5 & 10 & 18,5 & 0,543 \\
\hline Ampicilina & 3 & 20,0 & 0 & 0,0 & 3 & 5,9 & 0,022 \\
\hline Duración del tratamiento antimicrobiano $\left(\right.$ media, DE) ${ }^{* *}$ & 4,0 & 5,5 & 10,1 & 4,9 & 8,3 & 5,7 & $<0,001$ \\
\hline Indicación de fármacos vasoactivos & 13 & 92,9 & 12 & 32,4 & 25 & 49,0 & $<0,001$ \\
\hline Indicación de corticosteroides & 15 & 100,0 & 15 & 38,5 & 30 & 55,6 & $<0,001$ \\
\hline
\end{tabular}

bas $^{17}$. La meningococcemia es una infección fulminante (habitualmente menor a $24 \mathrm{~h}$ ) que suele presentarse en las primeras 4-6 h con síntomas inespecíficos, entre ellos fiebre, mialgias, coriza, odinofagia, anorexia, náuseas y/o vómitos, irritabilidad en pacientes bajo 5 años de edad y cefalea. Todos ellos son indistinguibles de los síntomas presentes habitualmente en las infecciones respiratorias virales ${ }^{18}$. En cambio, los clásicos síntomas (rash hemorrágico, signos meníngeos) suelen presentarse a las 13-22 h y a su vez, la aparición de un rash petequial y/o purpúrico se presenta también en sepsis bacterianas o virales, vasculitis, desórdenes plaquetarios, entre otras patologías y por tanto, es difícil plantear el diagnóstico de EM antes de la aparición de síntomas específicos ${ }^{18-20}$. Los cuadros de meningococcemia pueden evolucionar rápidamente con hipotensión arterial, disfunción multiorgánica, isquemia periférica, pérdida de extremidades y muerte, hasta en $40 \%$ dentro de las primeras $24-48 \mathrm{~h}^{18,19}$. En la población del presente estudio, el síntoma más común fue la fiebre referida en $95 \%$ de los casos y cuantificada u objetivada en $38,0^{\circ} \mathrm{C}$ o más en $65 \%$ de ellos. Le siguen en frecuencia el cuadro catarral respiratorio, náuseas y/o vómitos, anorexia y diarrea, siendo esta última más frecuente y con una diferencia estadísticamente significativa en los fallecidos. En cambio, los signos de irritación meníngea en la primera consulta se observaron con una baja frecuencia $(8,7 \%)$. No fue posible establecer la temporalidad de los síntomas; sin embargo, al analizar los síntomas y signos al momento de la admisión hospitalaria, se observó un cambio en el cuadro clínico, predominando el deterioro del estado general en gran parte de los casos $(80,4 \%)$, seguido por los síntomas respiratorios y la taquicardia. Por su parte, los signos de irritación meníngea presentaron una mayor frecuencia respecto al momento de la primera consulta (27,9 versus $8,7 \%)$. Otro hallazgo a destacar es la mayor presencia de rash petequial y/o purpúrico en los fallecidos, hecho que se debe muy probablemente a la mayor gravedad de estos casos. En este sentido, diversos estudios basados en brotes epidémicos, estiman que 28$77 \%$ de pacientes se presenta con rash al momento del ingreso hospitalario, en el caso de Chile se presentó en $20,4 \%{ }^{22}$. En definitiva, la presencia de síntomas inespecíficos al inicio de la enfermedad en los casos de EM por serogrupo W135 en Chile, coincide con los hallazgos descritos en la evidencia científica, hecho que puede llevar a un importante sub-diagnóstico (en esta población fue de $3,4 \%$ ), siendo un punto importante considerando la rápida progresión y la alta tasa de mortalidad de la $\mathrm{EM}^{4}$. En efecto, algunos autores han observado síntomas atípicos iniciales en pacientes con sepsis meningocóccica. Un equipo de investigadores llevó a cabo un estudio de caracterización clínica en niños y adolescentes con sepsis meningocóccica y, observaron que en $72 \%$ de ellos se presentaron tres síntomas de aparición temprana (con una media de ocho horas): dolor de extremidades inferiores, manos y pies fríos, color cutáneo anormal. Asimismo, relacionaron la ausencia de signos clásicos con la baja derivación a un centro hospitalario ( $51 \%$ en la muestra del estudio) ${ }^{18,21}$. En ninguno de los 60 casos del 2012 en Chile, se describió la presencia de estos síntomas; no obstante, sí hubo un predominio de sintomatología inespecífica en la mayoría de los pacientes, y la derivación a un centro de alta complejidad, en global, ocurrió en $65,5 \%$ del total.

Por otra parte, si bien las principales manifestaciones clínicas de la EM son la meningitis (30-60\%) y la meningococcemia $(20-30 \%)^{14,23-24}$, existen también otras condiciones menos comunes que incluyen: neumonía (5-15\% de los casos), artritis ( $2 \%$ ), otitis media ( $1 \%$ ) y epiglotitis $\left(\right.$ menos del 1\%) ${ }^{14}$. En este estudio, 83,3\% de los casos se presentó en la forma de meningococcemia (considerando la meningococcemia, la combinación meningitis y meningococcemia y el síndrome de Waterhouse-Friderichsen) y la meningitis aislada se presentó solamente en $15 \%$ de los casos. Además se detectó un caso que debutó como 
artritis, hecho que, según se ha escrito, estaría asociado al complejo clonal ST11 que es el circulante en Chile y en otros países de la región ${ }^{25}$.

La letalidad de la EM se relaciona con la frecuencia de formas fulminantes. En la ficha clínica se registran sólo dos casos con diagnóstico de purpura fulminans. En nuestro estudio se observa que de los casos con meningococcemia fallece $34,0 \%$ (17 de 50 casos) y $64,7 \%$ de los fallecidos muere dentro de las primeras $24 \mathrm{~h}$. Es posible que se hayan presentado más casos de purpura fulminans y que no estén registrados como tal.

La meningococcemia puede presentarse en individuos sanos, sin factores de riesgo conocidos. Existen, sin embargo, determinados factores que pueden aumentar la portación de $N$. meningitidis y también la probabilidad de enfermar; entre ellos se encuentran el hacinamiento, la exposición pasiva o activa al humo del tabaco, la presencia de infecciones respiratorias previas o concomitantes, la inmunodeficiencia (especialmente el déficit del complemento) entre otros factores ${ }^{26}$. En el presente estudio se observó mayor frecuencia de antecedentes cardiovasculares en los fallecidos; es necesario puntualizar que la metodología utilizada sólo considera la obtención de datos desde la ficha clínica y documentos relacionados, así como un análisis descriptivo y por tanto no es posible establecer una asociación entre antecedentes cardiovasculares y mayor probabilidad de morir. Así también se desconoce la eventual participación de otros factores de riesgo.

Dentro de las alteraciones de laboratorio observadas al inicio del cuadro, en el presente estudio destacó la mayor frecuencia en fallecidos de trombocitopenia, creatininemia elevada y leucopenia. En cuanto a la PCR inicial (considerando los tres primeros días desde que se inició el cuadro clínico), la mayoría de los casos presentó un resultado por sobre el valor de referencia $(95,8 \%)$, y a pesar de no haber una diferencia estadísticamente significativa entre fallecidos y sobrevivientes, el valor de la media fue elevado (171,5 mg/L). Estos hallazgos coinciden con algunos estudios publicados en la literatura científica que proponen factores de riesgo predictores de sepsis bacteriana: recuento anormal de leucocitos $\left(<5.000 / \mathrm{mm}^{3}\right.$ o $\left.>15.000 / \mathrm{mm}^{3}\right)$, trombocitopenia $\left(<100.000 / \mathrm{mm}^{3}\right)$ y $\mathrm{PCR}>5 \mathrm{mg} / \mathrm{L}^{27,28}$.

Del análisis de la evolución temporal de los casos, se observa que la primera consulta se realizó dentro de un plazo de 24-48 h desde que se inició el cuadro clínico, en $80,0 \%$ de los casos, y se realizó la hospitalización oportuna en $86,2 \%$ de los casos. Este último corresponde a un indicador de proceso de la calidad de la vigilancia, cuyo objetivo es disminuir la letalidad de la EM mediante tratamiento oportuno y obtención de muestra adecuada para la confirmación del caso. En ambos períodos, no se observaron diferencias entre sobrevivientes y fallecidos. Por su parte en los fallecidos se observó una rápida y fulminante evolución hacia el desenlace fatal, 70,6\% falleció dentro de los 3 primeros días de hospitalización y $63,2 \%$ falleció dentro de los primeros 4 días desde que se presentaron los primeros síntomas. Otro modo de valorar la rápida evolución en los fallecidos es la estadía hospitalaria, la cual fue significativamente menor en los fallecidos respecto a los sobrevivientes.

En los sobrevivientes no es infrecuente observar la presencia de secuelas; entre ellas, la hipoacusia y la necrosis de piel se encuentran dentro de las más comunes. En un estudio realizado por Kaplan y cols., se presentaron en $9,6 \%$ cada una, y las secuelas motoras con frecuencia de $4,9 \%{ }^{24}$. Las amputaciones son resultado de la necrosis cutánea, muscular y ósea de las extremidades afectadas. De los 60 casos registrados en el año 2012, las secuelas se presentaron en seis casos $(10,0 \%)$ : hipoacusia en dos, déficit motor en dos y necrosis con requerimiento de amputación y resolución quirúrgica en dos pacientes, uno de los cuales finalmente falleció.

El cuadro clínico inicial caracterizado por síntomas y signos inespecíficos, la presentación principal como meningococcemia y la rápida evolución de los casos podría explicar, en parte, la alta tasa de mortalidad observada en el aumento de casos de la EM por serogrupo W135 en el año 2012. Sin embargo, a partir del presente estudio no es posible determinar otros factores asociados a mayor letalidad además de las características clínicas. El futuro comportamiento de la EM en Chile es impredecible, sólo sabemos que permanecerá en la comunidad y todavía son muchas las interrogantes que quedan por responder, por ejemplo: ¿qué otros factores de riesgo o de exposición están influyendo en el aumento de casos y en el desenlace fatal de muchos de ellos? A partir de la información clínica y epidemiológica de la situación del año 2012, ¿es posible predecir el escenario del año 2013? ¿Se conoce la prevalencia de portación de $N$. meningitidis en Chile? Siendo la vacunación la medida de control de mayor eficacia, se deberá analizar la inclusión como vacuna programática en el Programa Nacional de Inmunizaciones $\mathrm{y}$, de acuerdo a los futuros estudios y el comportamiento epidemiológico, definir los grupos de edad a cubrir. Por otro lado, en Chile la EM según el Decreto $\mathrm{N}^{\circ} 158$ del año 2004, es de notificación universal e inmediata. La vigilancia epidemiológica ha sido eficiente y de calidad, la notificación de casos sospechosos de EM también ha sido oportuna y en el contexto de W135, esto ha permitido que la quimioprofilaxis se entregara a todos los contactos que la requerían, contribuyendo a evitar la aparición de casos secundarios. ${ }^{13}$ En el ámbito clínico, es importante que los profesionales médicos permanezcan atentos ante un paciente que presenta fiebre, rápido y abrupto deterioro del estado general junto a otros síntomas inespecíficos. Una vez planteado el diagnóstico, uno de los principales objetivos del tratamiento es el inicio rápido de la 
cobertura antimicrobiana. La experiencia publicada en otros países recomienda además, una rápida derivación y traslado desde un centro de baja complejidad a un centro hospitalario o de mayor complejidad e ingreso en UCI o unidades similares con el objetivo de mantener bajo estricta monitorización al paciente ${ }^{31,32}$.

\section{Resumen}

Introducción: En el año 2012 en Chile, se presentaron 60 casos de enfermedad meningocóccica (EM) causadas por serogrupo W135, que representa $57,7 \%$ de los casos seroagrupables. Objetivo: Describir las características clínicas de los casos de EM por serogrupo W135 confirmados durante el año 2012. Material y Métodos: Estudio descriptivo, de series de casos basada en la revisión de las fichas clínicas. Resultados: El 61,7\% de los casos fueron varones y 46,7\% tenía menos de 5 años. En la primera consulta, 3,4\% tuvo sospecha de EM, en tanto 83,3\% tuvo diagnóstico final de meningococcemia. En la primera consulta, los síntomas y/o signos más frecuentes fueron fiebre $\geq 38,0^{\circ} \mathrm{C}(60,3 \%)$, cuadro catarral respiratorio $(52,5 \%)$ y náuseas y/o vómitos (46,7\%). Mientras que los signos de irritación meníngea se presentaron en $8,7 \%$. En los fallecidos la diarrea fue el segundo síntoma más frecuente $(55,6 \%)$, y estadísticamente superior respecto de los sobrevivientes $(26,8 \% ; \mathrm{p}=0,034)$. Seis casos presentaron secuelas: amputaciones de extremidades, hipoacusia o daño neurológico y la letalidad fue de 31,7\%. Discusión: la EM por el serogrupo W135 en el año 2012, tuvo una elevada letalidad, presentación clínica inespecífica, sospecha diagnóstica inicial baja y un alto número de casos cursaron con una mala evolución.

\section{Referencias bibliográficas}

1.- Ministerio de Salud de Chile. Plan de acción W135. Disponible en: http://www.minsal.gob. $\mathrm{cl} /$ portal/url/page/minsalcl/g_varios/boletin/ meningitis/page_1.html. (consultado el 15 de febrero del 2013).

2.- Pollard A J. Global epidemiology of meningococcal disease and vaccine efficacy. Pediatr Infect Dis J 2004; 23 (12 Suppl): S274-9.

3.- World Health Organization. Background paper on meningococcal vaccines, WHO Strategic Advisory Group of Experts on Immunization, 2011.Disponible en: http:// www.who.int/ immunization/sage/1_mening_background document_v5_3_apr_2011.pdf. (consultado el 04 de febrero del 2013).

4.- Rosenstein N E, Perkins B A, Stephens D S, Popovic T, Hughes J M. Meningococcal disease. N Engl J Med 2001; 344 (18): 1378-88.

5.- Greenwood B. Manson Lecture. Meningococcal meningitis in Africa. Trans R Soc Trop Med Hyg 1999; 93 (4): 341-53.

6.- World Health Organization. Nota descriptiva No 141: Meningitis meningocócica, 2012. Disponible en: http:/www.who.int/mediacentre/ factsheets/fs141/es/index.html. (consultado el 16 de febrero del 2013).

7.- $\quad$ Palazzi M A, Cintra O A. Epidemiology of meningococcal disease in Latin America: current situation and opportunities for prevention. Neurol Res 2010; 32 (3): 263-71.

8.- Gallegos D. Enfermedad meningocócica: un cambio de presentación de baja endemia. Boletín de Vigilancia en Salud Pública de Chile. 2007; 10 (24): 18-21.

9.- Ministerio de Salud de Chile, Departamento de Epidemiología. Situación epidemiológica de la enfermedad meningocóccica, 30 de septiembre del 2012. Disponible en: http://www.sochipe.cl/ subidos/noticias2/docs/MeningococoMinsal.pdf. (consultado el 10 de febrero del 2013).

10.- Barroso D E, Rebelo M C. Recognition of the epidemiological significance of Neisseria meningitidis capsular serogroup W135 in the Rio de Janeiro region, Brazil. Mem Inst Oswaldo Cruz 2007; 102(6): 773-5.

11.- López E L, Debbag R. Meningococcal disease: always present. Serogroup changes in the Southern Cone. Rev Chilena Infectol 2012; 29 (6): 587-94.

12.- Ministerio de Salud de Chile, Departamento de Epidemiología. Enfermedad meningocócica. Situación epidemiológica, enero-marzo, 2013. Disponible en: http://epi.minsal.cl/epi/html/ AtlasInteractivos/AtlasBET/ABET_01/BET_1_ EM.pdf. (consultado el 27 de junio del 2013).

13.- Gallegos D, Maldonado A, Cáceres K, Seoane M. Situación epidemiológica y cumplimiento de indicadores de la vigilancia de la enfermedad meningocócica. Boletín de Vigilancia en Salud Pública de Chile 2012; 13 (27): 56-63.

14.- Bilukha O O, Rosenstein N; National Center for Infectious Diseases, Centers for Disease Control and Prevention (CDC). Prevention and control of meningococcal disease. Recommendations of the Advisory Committee on Immunization Practices (ACIP). MMWR Recomm Rep 2005; 54 (RR-7): 1-21.

15.- Ministerio de Salud de Chile, Subsecretaría de Salud Pública.Ley 20584: Regula los derechos y deberes que tienen las personas en relación con acciones vinculadas a su atención en salud. Publicada en el diario oficial el 24 de Abril del 2012. (consultado el 15 de diciembre del 2012).

16.- Ministerio de Salud de Chile, Departamento de Asesoría Jurídica. D.F.L. No 01 del año 2005:
Fija texto refundido, coordinado y sistematizado del decreto ley $\mathrm{N}^{\circ} 2763 / 79$ y de las leyes $\mathrm{N}^{\circ}$ S. 18.933 y N $\mathrm{N}^{\circ} 18.469$. Publicada en el diario oficial el 24 de Abril del 2006 (consultado el 15 de diciembre del 2012).

17.- Pace D, Pollard A J. Meningococcal disease: clinical presentation and sequelae. Vaccine 2012; 30 (30S): B3-9.

18.- Thompson M J, Ninis N, Perera R, Mayon-White R, Phillips C, Bailey L, et al. Clinical recognition of meningococcal disease in children and adolescents. Lancet. 2006; 367(9508): 397-403.

19.- Chang Q, Tzeng Y L, Stephens D S. Meningococcal disease: changes in epidemiology and prevention. Clin Epidemiol 2012; 4: 237-45.

20.- Wells L C, Smith J C, Weston V C, Collier J, Rutter N. The child with a non-blanching rash: how likely is meningococcal disease? Arch Dis Child. 2001; 85 (3): 218-22.

21.- Haj-Hassan T A, Thompson M J, Mayon-White R T, Ninis N, Harnden A, Smith L F, et al. Which early 'red flag' symptoms identify children with meningococcal disease in primary care? Br J Gen Pract 2011; 61 (584): e97-104.

22.- Stephens D S, Greenwood B, Brandtzaeg P. Epidemic meningitis, meningococcaemia, and Neisseria meningitidis. Lancet 2007; 369 (9580): 2196-210.

23.- European Union Invasive Bacterial Infections Surveillance Network: invasive Neisseria meningitidis in Europe-2006. EU-IBIS Network Company Publication; 2008, April.

24.- Kaplan S L, Schutze G E, Leake J A, Barson W J, Halasa N B, Byington C L, et al. Multicenter surveillance of invasive meningococcal infections in children. Pediatrics 2006; 118 (4): e979-84. 
25.- Vienne P, Ducos-Galand M, Guiyoule A, Pires R, Giorgini D, Taha M K, et al. The role of particular strains of Neisseria meningitidis in meningococcal arthritis, pericarditis, and pneumonia. Clin Infect Dis 2003; 15; 37 (12): 1639-42.

26.- Harrison L H, Kreiner C J, Shutt K A, Messonnier N E, O'Leary M, Stefonek K R, et al; Meningococcal High School Study Group. Risk factors for meningococcal disease in students in grades 9-12. Pediatr Infect Dis J 2008; 27 (3): 193-9.

27.- Brogan P A, Raffles A. The management of fever and petechiae: making sense of rash decisions. Arch Dis Child 2000; 83 (6): 506-7.

28.- Wong V K, Hitchcock W, Mason W H. Meningococcal infections in children: a review of 100 cases. Pediatr Infect Dis J 1989; 8 (4): 224-7.

29.- Pelton S I. Meningococcal disease awareness: clinical and epidemiological factors affecting prevention and management in adolescents. J Adolesc Health 2010 (46): S9-S15.

30.- Tunkel A R, Hartman B J, Kaplan S L, Kaufman B A, Roos K L, Scheld W M, et al.
Practice guidelines for the management of bacterial meningitis. Clin Infect Dis 2004; 39 (9): 1267-84.

31.- Goldacre M J, Roberts S E, Yeates D. Case fatality rates for meningococcal disease in an English population, 1963-98: database study. Br Med J 2003; 327 (7415): 596-7.

32.- Booy R, Habibi P, Nadel S, de Munter C, Britto J, Morrison A, et al; Meningococcal Research Group. Reduction in case fatality rate from meningococcal disease associated with improved healthcare delivery. Arch Dis Child 2001; 85 (5): 386-90 